\title{
A velocity decomposition formulation for 2D steady incompressible lifting problems
}

\author{
William J. Rosemurgy, Robert F. Beck, Kevin J. Maki* \\ Department of Naval Architecture and Marine Engineering \\ University of Michigan \\ 2600 Draper $R d$. \\ Ann Arbor, MI 48109-2145 USA
}

\begin{abstract}
The principle of velocity decomposition is used to efficiently and accurately solve the Navier-Stokes boundary-value problem for lifting flows. The velocity vector is decomposed as the sum of irrotational and vortical components. The irrotational component is represented using a velocity potential which satisfies the Laplace equation with a modified boundary condition that is written in terms of the Navier-Stokes solution. A viscous potential can be found which satisfies the Navier-Stokes problem directly outside of the rotational regions of the fluid such that the fluid domain over which the Navier-Stokes equations must be solved numerically can be greatly reduced. The viscous potential is used as a Dirichlet boundary condition for the total velocity on the boundaries of the reduced fluid domain. The velocity decomposition approach is used to solve for the flow over a 2D NACA0012 foil in both laminar and turbulent regimes.

Keywords: Incompressible Navier-Stokes flow, Velocity Decomposition, Viscous potential flow, Viscous-Inviscid interaction
\end{abstract}

\section{Introduction}

In this paper we present a theoretical framework that uses a functional decomposition of the velocity vector to solve the Navier-Stokes boundary-value

* Corresponding author
Email address: kjmaki@umich.edu (Kevin J. Maki)

Preprint submitted to European Journal of Mechanics - B/Fluids

March 19, 2016

(c) 2016. This manuscript version is made available under the Elsevier user license http://www.elsevier.com/open-access/userlicense/1.0/ 
problem. Typical numerical strategies for solution of the Navier-Stokes prob5 lem for external flows require discretization of relatively large fluid domains. In this work the discretized domain can be substantially reduced to encompass the region where the fluid velocity has rotation. Specifically, we continue the development from [1] which treated steady, incompressible, axisymmetric, external flows to include problems where lift (circulation) is present in the flow.

It is a well-known characteristic that for flows of sufficiently high Reynolds number, e.g. $\operatorname{Re} \geq 10^{3}$, the region where the vorticity vector is non-zero is much smaller than the region where the velocity field is affected by the body. Generally, vorticity in the fluid is generated at the body boundary due to the no-slip boundary condition, and diffuses slowly into the domain due to the small diffusion coefficient (viscosity). The vorticity is convected downstream or dissipates quickly with respect to the upstream or perpendicular distance from the boundary. Therefore, the vast majority of the fluid is irrotational in nature. In the irrotational regions of the flow, a scalar velocity potential can be used to represent the solution to the Navier-Stokes problem.

The Navier-Stokes equations are the governing equations of motion for the real fluid flow. However, they are a set of nonlinear and fully coupled equations while the Laplace equation for the velocity potential is a linear equation with a single unknown. Generally, the numerical solution of the Navier-Stokes boundary-value problem using a field method can require a much greater com25 putational effort than solving the Laplace equation using a boundary-element method. There is a significant computational cost advantage to solving the Laplace equation for the velocity potential over the Navier-Stokes equations for the velocity vector and pressure fields.

Many researchers have devised methods to take advantage of the fact that the majority of the fluid flow is irrotational in most real-world external flow problems. One common approach involves decomposing the Navier-Stokes boundaryvalue problem by decomposing the working variables (velocity or vorticity) into different components and solving for each using the most efficient method. Another decomposition method involves splitting the computational domain 
into sub-domains (for example: near the body, downstream of the body, and the far-field) and applying appropriate solution techniques to each sub-domain [2, 3, 4, 5, 6]. Finally, and possibly most simply, many approaches have been developed to modify the velocity potential to include the effects of vorticity.

A straightforward technique to include viscous effects in a velocity potential

40 approach is found in [7]. In this paper, Lighthill derives four different approaches to include the concept of displacement thickness into a velocity potential formulation. 8] solves the 3D velocity potential with a displacement thickness approximation calculated from the 2D boundary layer equations. XFOIL [9], a well-known airfoil analysis and design tool, uses the "equivalent source" ap45 proach from [7] to capture viscous effects.

In [10, 11, 12, 13, 14, Morino and his colleagues extensively investigate the use of velocity decomposition using the Helmholtz decomposition to solve external flow problems with lift. In all but the latest work, the vorticity equations are used to solve for the rotational component of the flow. We use [10] as the theoretical motivation for portions of the work presented here. In our work, we always advocate for the use of the momentum equations in the primitive variables of velocity and pressure because there are a broad range of numerical techniques available to solve the momentum equations. Furthermore and most importantly, the body boundary condition on the velocity vector is trivial in the momentum equations, whereas in the vorticity equation the body boundary condition is not straightforward.

In [15, 16, 17, the authors also employ the Helmholtz decomposition which is substituted into the momentum equations. The velocity potential is found by solving a Poisson equation with a source term related to the vortical velocity. boundary-element method because it can be more computationally efficient and a BEM provides the solution in the entire free-space, not just in the region of the field discretization.

Finally, an investigation into the relationship between potential flow and ${ }_{65}$ irrotational viscous flow is given in [18, 19, 20]. These papers provide fun- 
damental discussion on the subtle difference between a velocity potential that solves inviscid fluid problems versus those that solve viscous fluid problems.

The ultimate goal of the present work is the solution of problems of bodies in waves. 21] uses a functional decomposition of the Navier-Stokes equations 70 to study water wave mechanics.

In the velocity decomposition used in this work, the velocity vector is decomposed using a Helmholtz-like decomposition into to an irrotational (curl-free) component and a solenoidal (divergence-free) component, Eq. (1).

$$
\mathbf{u}=\nabla \varphi+\mathbf{w}
$$

Where $\mathbf{u}$ is the total fluid velocity and satisfies the Navier-Stokes problem, $\nabla \varphi$ is the the irrotational component and is modeled by a velocity potential, and $\mathbf{w}$ is the rotational component of the velocity. The region over which the rotational flow is solved is reduced to the regions only where vorticity is present $(|\boldsymbol{\omega}| \neq 0$ 75 and $|\mathbf{w}| \neq 0)$. The velocity potential is used to specify the Dirichlet condition for the total velocity on the boundaries of the reduced domain which are placed just outside the vortical regions of the flow $(|\boldsymbol{\omega}|=0$ and $|\mathbf{w}|=0)$.

In order to fully realize the advantages of a velocity decomposition approach to solving viscous flows, the velocity potential must satisfy the Navier-Stokes so equations directly outside of the vortical regions of the flow. This is not the case for the velocity potential which satisfies conventional boundary conditions. Therefore, the velocity potential boundary-value problem must be modified. In this work, the velocity decomposition approach will be developed for application to lifting problems and the modifications to the velocity potential in order to solve these problems will be discussed in full.

The current research is based off of the work in 22] and 23]. Similar to the methods above, in [22] the velocity vector is decomposed into an irrotational (potential) velocity component and a vortical velocity term. The decomposition is substituted into the RANS equations and, after mathematical reduction,

90 the "complementary RANS" equations are derived. This new set of governing equations is solved throughout the fluid domain. The method was shown to 
reproduce the accuracy of traditional RANS simulations but did not exhibit a significant computational speed-up. A reduction in domain size was not realized because the potential solution was not adjusted to ensure compatibility with the viscous solution after the initial guess for the potential solution was calculated.

In [24], improvements were made to the original work of [22] by introducing a transpiration velocity term to the body boundary condition which improves the agreement between the inviscid and viscous solutions. The complementary RANS equations were solved using this improved velocity potential and a slight improvement in the solution time was realized. This approach, however, was discarded in favor of a more direct approach.

In 23], the velocity decomposition is only applied to the body- and far-fieldboundary conditions. The far-field boundary of a reduced Navier-Stokes domain is located where the vortical component of velocity is zero and the velocity potential fully describes the flow. In order to achieve this, a non-homogeneous body-boundary condition is derived by applying the velocity decomposition to the body-boundary condition, following [10. The velocity potential which satisfies this modified body-boundary condition satisfies the Navier-Stokes equations directly outside of the vortical region of the flow. This allows for the NavierStokes equations to be explicited solved only in the vortical region. The result of this is an accurate and computationally efficient solution method which is able to solve for the viscous flow around a body in fractions of the time required by a conventional Navier-Stokes approach.

In the rest of this paper we will discuss the extension of the velocity decom115 postion approach to include two-dimensional problems with lift. The theoretical framework discussed in this paper is numerically implemented to solve the Navier-Stokes boundary-value problem in a computationally efficient manner using velocity decomposition. The approach is used to solve for the laminar and turbulent flow over a two-dimensional NACA0012 airfoil. 


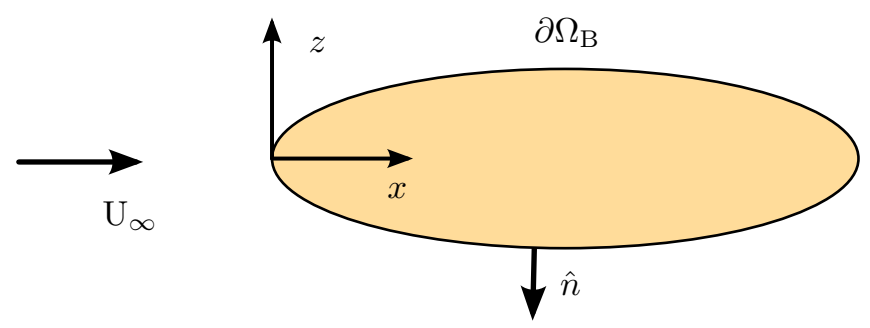

Figure 1: The definition of the Navier-Stokes boundary value problem.

\section{Navier-Stokes Boundary Value Problem}

The types of flows of interest in this research are those that satisfy the Navier-Stokes boundary-value problem. Specifically, the Navier-Stokes problem is restricted to steady, incompressible flow equations. Furthermore, all work presented in this paper is performed in two-dimensions (in [1, the approach is applied to three-dimensional axisymmetric flows). As shown in Fig. 1, the coordinate system is body fixed and the position vector is expressed as:

$$
\mathbf{x}=x \hat{\mathbf{i}}+z \hat{\mathbf{k}}
$$

The Navier-Stokes equations consist of the conservation of mass and the conservation of linear momentum and are given in Eqs. (3) and (4). The fluid domain and the boundaries are shown in Fig. 1

$$
\begin{aligned}
\nabla \cdot \mathbf{u} & =0 \\
\nabla \cdot \mathbf{u} \otimes \mathbf{u} & =-\nabla p / \rho+\nabla \cdot \nu\left(\nabla \otimes \mathbf{u}+\nabla \otimes \mathbf{u}^{\mathrm{T}}\right)
\end{aligned}
$$

Where $\mathbf{u}$ is the velocity vector defined in Eq. (5), $p$ is the pressure, $\rho$ is the fluid density, $\nu$ is the kinematic viscosity of the fluid, and ()$^{\mathrm{T}}$ is the transpose.

$$
\mathbf{u}=u \hat{\mathbf{i}}+w \hat{\mathbf{k}}
$$

The Navier-Stokes boundary-value problem also requires the specification of boundary conditions in order to be well posed. Eq. (6) is the no-slip body boundary condition and is applied to $\partial \Omega_{\mathrm{B}}$, as shown in Fig. 1.

$$
\mathbf{u}=0 \quad \text { on } \quad \partial \Omega_{\mathrm{B}}
$$


Eq. (7) is the far-field boundary condition.

$$
\lim _{|\mathbf{x}| \rightarrow \infty} \mathbf{u}=\mathrm{U}_{\infty} \hat{\mathbf{i}}
$$

It is assumed throughout this paper that the term "Navier-Stokes equations" can also refer to the Reynolds-averaged Navier-Stokes (RANS) equations. For low Reynolds number cases we solve the steady Navier-Stokes equations. For high $\operatorname{Re}$ flows $\left(\operatorname{Re}>10^{6}\right)$ we solve the steady Reynolds-averaged equations and use the $k-\omega$ SST turbulence model to approximate the eddy-viscosity.

\section{Velocity Decomposition}

Now that the Navier-Stokes boundary-value problem has been defined, the velocity decomposition approach used to solve it can be described. The decomposition of the velocity vector is based on the fundamental theorem of vector calculus (Helmholtz decomposition) which states that any vector field can be expressed as the sum of an irrotational (curl-free) field and a solenoidal (divergence-free) field [25]. Eq. (8) defines the notation used in this work.

$$
\mathbf{u}=\nabla \varphi+\mathbf{w}
$$

Where the irrotational component of the velocity decomposition is represented as the gradient of a scalar, $\varphi$, and the solenoidal component is $\mathbf{w}$ is also referred to as the vortical component.

The strategy motivating the use of velocity decomposition for Navier-Stokes problems is to reduce the size of the flow domain over which a Navier-Stokes solver must be used by representing the majority of the flow field with a velocity potential. The velocity potential is then used to supply boundary conditions to the Navier-Stokes solver. This is illustrated in Fig. 2 and Eq. (9), where $\partial \Omega_{\mathrm{E}}$ is the boundary of the reduced Navier-Stokes domain.

$$
\mathbf{u}=\nabla \varphi \quad \text { on } \quad \partial \Omega_{\mathrm{E}}
$$

The decomposition stated in Eq. (8) is not unique; there are an infinite number of combinations of vector fields which could combine to re-create the total 


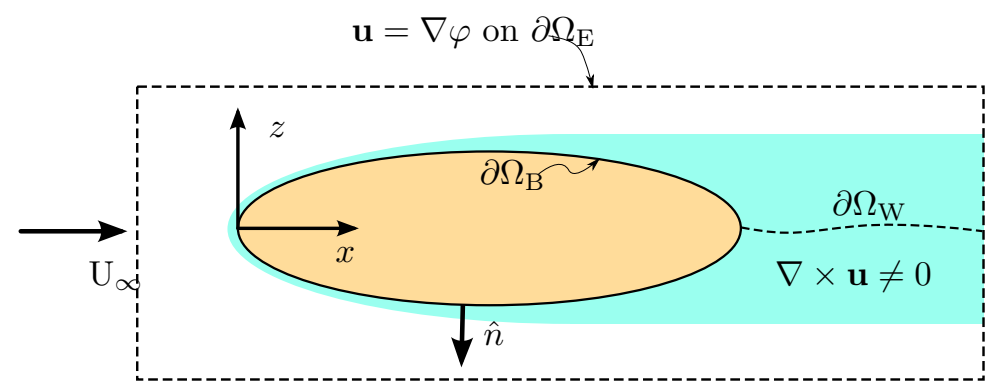

Figure 2: A schematic of the decomposed Navier-Stokes Problem.

velocity field. Therefore, a condition is enforced on the decomposition to require the vortical component, w, to go to zero outside of the vortical region of the flow. To put it another way, as the distance from the body grows, the vortical component in the velocity decomposition goes to zero. Once the vortical component is negligible, the total velocity vector can be represented in full by the velocity potential. It is therefore essential to use a velocity potential which satisfies the Navier-Stokes problem directly outside of the vortical regions of the flow. Unfortunately, the velocity potential which satisfies the conventional inviscid boundary conditions on the body - non-penetration and, in lifting flows, smooth flow at the trailing edge - does not satisfy this requirement. The potential that satisfies the inviscid body boundary condition is denoted $\Phi$. This is very clearly shown in Fig. 3 where the streamwise component of the potential velocity is compared to the streamwise component of the viscous flow over a NACA0012 foil at $R e=2000$ at an angle of attack, $\alpha=5^{\circ}$. In fact, the velocity potential does not satisfy the Navier-Stokes problem even at large distances from the body. Additionally, the lift coefficient determined from the velocity potential does not capture the loss of lift due to separation and stall present in the viscous solution.

Therefore, a different velocity potential, the viscous potential, must be introduced. The viscous potential is a velocity potential which satisfies the NavierStokes problem directly outside of the vortical region of the flow. In the current approach, the viscous potential is represented as $\varphi$ and satisfies a non- 


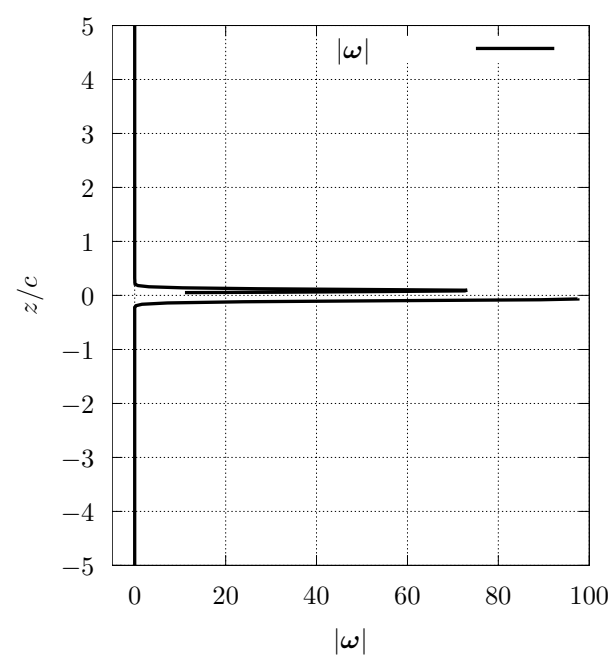

(a) Magnitude of vorticity at the midchord

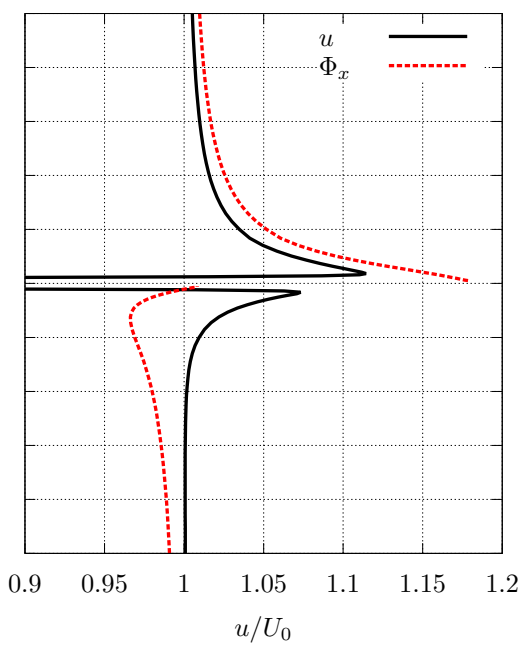

(b) Streamwise velocity at the midchord

Figure 3: Vorticity and streamwise velocity over a sample line at the midchord of the NACA0012 foil at $\operatorname{Re}=2000$.

homogeneous body-boundary condition. Recall that the inviscid potential $\Phi$, is the velocity potential which satisfies the conventional non-penetration bodyboundary condition. Eqs. 10], 11], and (12) form the basis of the velocity decomposition using the viscous potential.

$$
\begin{aligned}
& \mathbf{u}=\nabla \varphi+\mathbf{w} \\
& \mathbf{u}=\nabla \varphi \quad \text { on } \quad \partial \Omega_{\mathrm{E}} \\
& \frac{\partial \varphi}{\partial n}=f(\mathbf{x}, \mathbf{u}) \quad \text { on } \quad \partial \Omega_{\mathrm{B}}
\end{aligned}
$$

Now that the general velocity decomposition approach has been introduced, the Navier-Stokes problem described in Section 2 will be decomposed into the Navier-Stokes sub-problem (Section 3.1) and the viscous potential sub-problem (Section 3.2).

Throughout this paper, the term velocity decomposition refers to the theoretical and numerical approach of solving the Navier-Stokes and viscous potential sub-problems in order to achieve the solution of the full Navier-Stokes 
boundary-value problem. While the term viscous potential specifically refers to the velocity potential which satisfies the Navier-Stokes problem outside of the vortical regions of the flow.

\subsection{Navier-Stokes Sub-Problem}

The Navier-Stokes sub-problem is defined as the Navier-Stokes equations Eqs. (3), (4), and (10), on a reduced domain. The reduced domain extends from the body boundary $\partial \Omega_{\mathrm{B}}$ to $\partial \Omega_{\mathrm{E}}$ (see Fig. 2 ).

$$
\begin{aligned}
\nabla \cdot \mathbf{u} & =0 \\
\nabla \cdot \mathbf{u} \otimes \mathbf{u} & =-\nabla p / \rho+\nabla \cdot \nu\left(\nabla \otimes \mathbf{u}+\nabla \otimes \mathbf{u}^{\mathrm{T}}\right) \\
\mathbf{u} & =\nabla \varphi+\mathbf{w}
\end{aligned}
$$

The no-slip body boundary condition, 6, is still enforced. The important difference between the Navier-Stokes problem and the Navier-Stokes sub-problem is that the domain of the sub-problem is drastically reduced to only include the region of the flow which is vortical. The boundary condition on the boundary of the reduced domain, $\partial \Omega_{\mathrm{E}}$, is given in Eq. (13).

$$
\mathbf{u}=\nabla \varphi \quad \text { on } \quad \partial \Omega_{\mathrm{E}}
$$

Pressure boundary conditions are included as part of the numerical approach and discussed in Section 4.2 .

\subsection{Viscous Potential Sub-Problem}

The irrotational component of the velocity is represented using a velocity potential. If the flow field is irrotational, then the velocity field can be represented by the gradient of a scalar field, Eq. (14) 25].

$$
\mathbf{u}=\nabla \varphi
$$

Eq. (14) is substituted into the continuity equation, Eq. (3), which results in the Laplace equation (Eq. 15) that governs the velocity potential. Eqs. 15 
and 10 are the governing equations for the viscous potential sub-problem.

$$
\begin{aligned}
\nabla^{2} \varphi & =0 \\
\mathbf{u} & =\nabla \varphi+\mathbf{w}
\end{aligned}
$$

The governing Laplace equation for the viscous potential is solved using the conventional boundary-element method widely discussed in the literature ([26, for example). Green's second identity is used to express the velocity potential as a surface distribution of singularities which are fundamental solutions to Laplace's equation. Therefore, the velocity potential is represented by distributions of sources and dipoles on the boundaries of the problem as shown in Eq. (16).

$$
\varphi=\int_{\partial \Omega_{\mathrm{B}}+\partial \Omega_{\mathrm{W}}}\left[\sigma \ln r+\mu \frac{\partial}{\partial n}(\ln r)\right] \mathrm{d} \mathrm{S}+U_{\infty} x
$$

Where $\sigma$ and $\mu$ are the unknown strengths of the source and dipole distributions and the boundaries are defined in Fig. 2. The strengths of the singularities are determined by solving a linear system based on the application of boundary conditions at collocation points. However, there are twice as many unknowns as there are boundaries (and boundary conditions). Therefore, additional constraints or assumptions must be stated in order to make the problem unique. For example, some solution techniques assume that the strength of the source distribution is a known quantity and solve for the dipole strength while other approaches will set $\mu=0$ on the body, effectively removing the dipole distribution from the body. In this work, the velocity potential is represented by a source and vortex (another fundamental solution) distribution on the body, and a source and dipole distribution on the wake surface.

In order to complete the viscous potential sub-problem, the boundary conditions for the viscous potential are now stated. Following [23], the body-boundary condition for the viscous potential is developed from the no-slip boundary con- 
dition, Eq. (6).

$$
\begin{aligned}
\mathbf{u} & =0 \quad \text { on } \quad \partial \Omega_{\mathrm{B}} \\
& =\nabla \varphi+\mathbf{w} \\
& \text { or } \\
\nabla \varphi & =-\mathbf{w} \quad \text { on } \quad \partial \Omega_{\mathrm{B}}
\end{aligned}
$$

The wall-normal component of Eq. (17) is the Neumann condition for the viscous potential, Eq. (18). The body-boundary condition for the viscous potential in Eq. 18 is written in terms of the vortical velocity. Note that the normal component of the potential gradient is non-zero on the body. This is what differentiates the viscous potential from the inviscid potential. The body-boundary condition for the viscous potential will be further developed in the next subsection.

$$
\frac{\partial \varphi}{\partial n}=-\mathbf{w} \cdot \hat{n} \quad \text { on } \quad \partial \Omega_{\mathrm{B}}
$$

In many flows of interest in this research, a large portion of the rotational flow exists in the region immediately downstream of the body in the viscous wake. It is important to include the influence of the rotational flow downstream of the body [23, 1]. The body-boundary condition for the viscous potential is also applied to the wake surface as shown in Eq. 19.

$$
\frac{\partial \varphi}{\partial n}=-\mathbf{w} \cdot \hat{n} \quad \text { on } \quad \partial \Omega_{\mathrm{W}}
$$

The role and importance of this condition is discussed in more detail in the results section.

The body-boundary condition for the viscous potential is not the conventional non-penetration condition. In fact, it requires that a flow exist through the body surface. The effect of the body-boundary condition for the viscous potential is to alter the shape of the body based on the thickness of the boundary layer and other viscous effects, such as separation. It is important to note that this approach has similarities to the displacement thickness technique from [7]. While there is some likeness in the approach, Lighthill's formulation makes the 


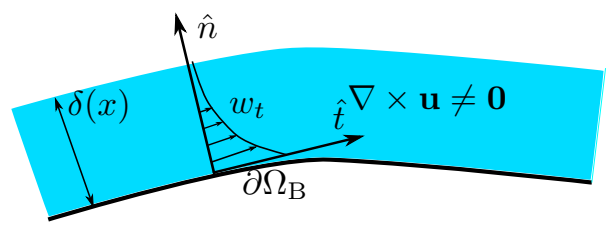

Figure 4: A figure describing the local-orthogonal coordinate system and the $\delta$-boundary.

185 hand, the approach used in this work is valid for cases where the boundary layer is very thick and massively separated (as in the case of the circular cylinder at $\operatorname{Re}=200$ ) and is directly applicable to three-dimensional situations as demonstrated in 23 .

The far-field boundary condition for the viscous potential is found by applying Eq. 10 to the far-field boundary condition for the Navier-Stokes subproblem. The vortical velocity goes to zero in the far field and the viscous potential fully describes the velocity field. Eq. 20) is the far-field boundary condition.

$$
\begin{aligned}
\mathbf{u} & =\nabla \varphi+\mathbf{w} \\
\lim _{|\mathbf{x}| \rightarrow \infty} \nabla \varphi & =\mathrm{U}_{\infty} \hat{\mathbf{i}}
\end{aligned}
$$

190

The far-field boundary conditions are automatically satisfied due to the nature of the singularities which are distributed on the body boundary.

\subsubsection{Body-boundary condition for the viscous potential}

The body-boundary condition for the viscous flow is motivated from [13] and directly follows [23], and is reproduced here for completeness. The divergencefree constraint on the total velocity vector also applies to the vortical velocity under the condition that the potential satisfies the Laplace equation. The divergnce-free constraint is expressed in a local-orthogonal coordinate system as shown in Fig. 4 .

$$
\frac{\partial w_{n}}{\partial n}+\frac{\partial w_{t}}{\partial t}=0
$$


Eq. (21) is then integrated along a line parallel to the local normal vector beginning at the body with length $\delta$. Where $\delta$ is defined as the distance in the normal direction from the body to the edge of the vortical region. The integral for the normal component of the vortical velocity is directly evaluated. The result is an expression for the normal component of the vortical velocity on the body, Eq. 222.

$$
\begin{aligned}
\frac{\partial w_{n}}{\partial n} & =-\frac{\partial w_{t}}{\partial t} \\
\int_{0}^{\delta} \frac{\partial w_{n}}{\partial n} \mathrm{dn} & =-\int_{0}^{\delta} \frac{\partial w_{t}}{\partial t} \mathrm{dn} \\
w_{n}(\delta)-w_{n}(0) & =-\int_{0}^{\delta} \frac{\partial w_{t}}{\partial t} \mathrm{dn} \\
w_{n}(0) & =\int_{0}^{\delta} \frac{\partial w_{t}}{\partial t} \mathrm{dn}+w_{n}(\delta)
\end{aligned}
$$

Then, Eq. (18) is used to obtain the Neumann condition for the viscous potential on the body by applying the requirement that the vortical velocity go to zero outside of the vortical region.

$$
\begin{aligned}
\frac{\partial \varphi}{\partial n} & =-\mathbf{w} \cdot \hat{n} \quad \text { on } \quad \partial \Omega_{\mathrm{B}} \text { and } \partial \Omega_{\mathrm{W}} \\
& =-w_{n}(0) \\
& =-\int_{0}^{\delta} \frac{\partial w_{t}}{\partial t} \mathrm{dn}-w_{n}(\delta){ }^{0} \\
\frac{\partial \varphi}{\partial n} & =-\int_{0}^{\delta} \frac{\partial w_{t}}{\partial t} \mathrm{dn} \quad \text { on } \quad \partial \Omega_{\mathrm{B}} \text { and } \partial \Omega_{\mathrm{W}}
\end{aligned}
$$

\subsubsection{Circulation condition for the viscous potential}

In the potential flow sub-problem the Kutta condition is used to ensure that a stagnation point exists at the trailing edge of a lifting body. When the Kutta condition is satisfied, the velocity field is smooth as it leaves the trailing edge. The Kutta condition can be stated in various ways; requiring that the velocity 
field be smooth as it leaves the trailing edge, requiring that a stagnation point be located at the trailing edge, or alternatively requiring zero pressure difference across a wake surface emanating from the trailing edge. Essentially, the Kutta condition is an additional physical constraint on the velocity potential which is used to determine the value of the body-bound circulation.

In this work, the equal pressure Kutta condition is used for the calculation of the inviscid potential. Eq. (24) is the result of using Bernoulli's equation to equate the pressure at two points where the small change in hydrostatic pressure is neglected. The two points are located just behind the trailing edge, slightly above and below the wake surface.

$$
\nabla \varphi^{+} \cdot \nabla \varphi^{+}-\nabla \varphi^{-} \cdot \nabla \varphi^{-}=0
$$

However, the issue with using a Kutta condition in the velocity decomposition framework is that it does not account for the loss of lift due to separation. Preliminary velocity decomposition calculations in 27] found that the lift coefficient was greatly over predicted when compared to the lift predicted by a Navier-Stokes solver. This was due to the fact that the viscous potential satisfied the inviscid Kutta condition, which resulted in a body-bound circulation that corresponded to a lift coefficient nearly twice the value of the lift coefficient predicted by a Navier-Stokes solver.

The solution to the problem of over-prediction of lift is to use the KuttaJoukowski theorem, Eq. 25), to relate the lift on the body calculated in the Navier-Stokes sub-problem to the total body-bound circulation, $\Gamma$, in the viscous potential sub-problem.

$$
\mathcal{L}=\rho \mathrm{U}_{\infty} \Gamma
$$

The force on the body in the Navier-Stokes sub-problem is calculated as the integral of the stress on the body as shown in Eq. 26). The lift is the component 
of the force in the $\hat{\mathbf{k}}$ direction, Eq. 27 .

$$
\begin{aligned}
\mathcal{F} & =\int_{\partial \Omega_{\mathrm{B}}}\left[p \mathbf{I}-\mu\left(\nabla \otimes \mathbf{u}+\nabla \otimes \mathbf{u}^{\mathrm{T}}\right)\right] \cdot \hat{n} \mathrm{~d} S \\
\mathcal{L}^{N S} & =\mathcal{F} \cdot \hat{\mathbf{k}}
\end{aligned}
$$

Once the lift from the Navier-Stokes sub-problem is calculated, the circulation required to generate this lift is found by re-arranging the Kutta-Joukowski theorem, Eq. 28).

$$
\Gamma^{N S}=\frac{\mathcal{L}^{N S}}{\rho \mathrm{U}_{\infty}}
$$

Finally, Eq. 29 is the total circulation condition which is used in place of the Kutta condition to determine the magnitude of the body-bound circulation in the viscous potential.

$$
\int_{\partial \Omega_{\mathrm{B}}} \gamma \mathrm{d} \mathrm{S}=\Gamma^{N S}
$$

\subsubsection{Source and dipole distribution on the wake surface}

The body-boundary condition for the viscous potential is also applied to the wake surface. One of the defining characteristics of a lifting flow is an asymmetric wake (with respect to the free-stream velocity vector). For example, Fig. 5 shows streamlines and contours of the magnitude of velocity of the flow over a NACA0012 foil at $\alpha=5^{\circ}$ with $\operatorname{Re}=2000$. Especially near the trailing edge, it is clear that the wake above a dividing streamline beginning from the trailing edge is not equal to the wake below it. This asymmetry must be accounted for in the viscous potential. The proposed solution to this problem is to use source and dipole panels coincidentally placed on the wake surface.

It is necessary to state the boundary condition that is satisfied on the wake surface. To simplify the discussion, the boundary condition will be developed for the case where only the wake source and dipole distributions are present. The effect of the body singularity distributions is included after the wake boundary condition has been described.

The body-boundary condition for the viscous potential requires an integration of a partial derivative of the vortical velocity over a line parallel to the body 


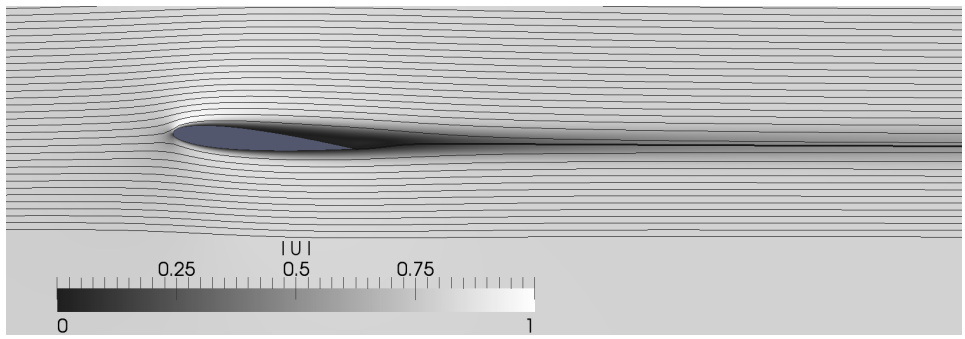

Figure 5: Streamlines of the viscous flow overlaid on contours of the magnitude of velocity field over a NACA0012 foil at $\mathrm{Re}=200$. The streamline emanating from the trailing edge is emphasized.

normal vector. Since the wake surface has two normal vectors pointing into the fluid (up and down), there are two boundary conditions that must be satisfied on the wake surface at the same place. The values of the boundary condition resulting from the integration over the upwards and downwards pointing normal vectors are denoted $\chi^{+}$and $\chi^{-}$, respectively. This presents a problem because there are two different conditions on the normal velocity that must be enforced simultaneously at the same location. In this work the wake source strength is determined using the average of the boundary conditions, and the wake dipole strength is set according to the difference. The conditions are stated in Eqs. (30) and (31) and shown in Fig. 6.

$$
\begin{aligned}
& \frac{\partial \phi_{W S}}{\partial n}=\frac{\chi^{+}+\chi^{-}}{2} \quad \text { on } \quad \partial \Omega_{\mathrm{W}} \\
& \frac{\partial \phi_{D}}{\partial n}=-\mathrm{U}_{\infty} \hat{\mathbf{i}} \cdot \hat{n}+\frac{\chi^{+}-\chi^{-}}{2} \quad \text { on } \quad \partial \Omega_{\mathrm{W}}
\end{aligned}
$$

Where $\phi_{W S}$ and $\phi_{D}$ represent the velocity potential due to the wake source and dipole distributions, respectively. Physically this means that the source panel is accounting for the thickness of the wake and the dipole panel is representing the asymmetry in the wake. The source and dipole combination is especially suited for this purpose due to the nature of their influence coefficients which describe the nature of the flow induced by each panel. As seen in Fig. 6, the source panel creates a jump in the normal velocity at the panel center while the dipole panel allows for a continuous normal flow through the panel. 


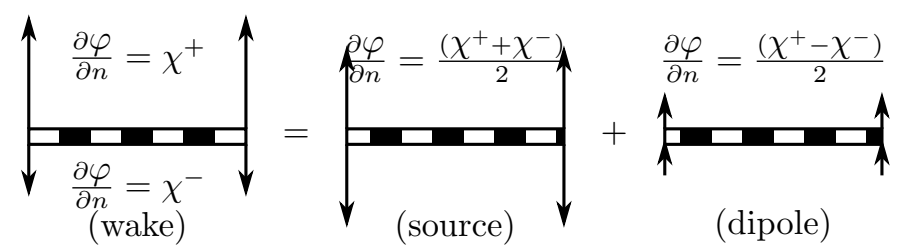

Figure 6: The asymmetry in the flow field in the wake results in different values of $\chi$ above and below the wake surface; a source panel is used to model the average, while a dipole panel accounts for the asymmetry.

\section{Numerical Implementation}

\subsection{Iterative Solution Strategy}

As described in Section 3 the interaction between the viscous potential and the Navier-Stokes sub-problems occurs on the body boundary, wake surface, and the boundary of the reduced domain, $\partial \Omega_{\mathrm{E}}$, through Eqs. 13 and 23 .

$$
\begin{aligned}
\mathbf{u} & =\nabla \varphi & & \text { on } \\
\frac{\partial \varphi}{\partial n} & =-\int_{0}^{\delta} \frac{\partial w_{t}}{\partial t} \mathrm{dn} & & \text { on }
\end{aligned}
$$

Eq. (23) provides the interaction from the Navier-Stokes sub-problem to the viscous potential sub-problem. Eq. 13 provides the interaction from the viscous potential sub-problem to the Navier-Stokes sub-problem. The two sub-problems are coupled and either need to be solved in a fully coupled manner or using an iterative scheme. An iterative approach is more fitting to this problem, mainly because the Navier-Stokes equations in the Navier-Stokes sub-problem are also solved in an iterative manner.

The first step in the iterative scheme is to initialize the Dirichlet condition for total velocity on $\partial \Omega_{\mathrm{E}}$ to the inviscid velocity potential, Eq. 32 .

$$
\mathbf{u}^{0}=\nabla \Phi \quad \text { on } \quad \partial \Omega_{\mathrm{E}}
$$

The Navier-Stokes equations are then solved iteratively using the SIMPLE algorithm, as described in Section 4.2, until the system of equations is partially 
converged. Once the equations reach partial convergence, the body-boundary condition for the viscous potential is computed from the viscous flow. The viscous potential is then calculated and the Dirichlet condition for the total velocity is updated, Eq. 33.

$$
\mathbf{u}^{i}=\nabla \varphi^{i} \quad \text { on } \quad \partial \Omega_{\mathrm{E}}
$$

Where the superscript $i$ is the velocity decomposition update number. In most cases, three to five velocity decomposition updates are needed.

245

\subsection{Navier-Stokes Sub-Problem}

The governing equations which describe the Navier-Stokes sub-problem are coupled, nonlinear, and the boundary conditions are dependent on the viscous potential. Analytical solutions are available for only a handful of canonical problems and a numerical strategy is employed to seek approximate solutions the Navier-Stokes sub-problem is also described in this section.

The Navier-Stokes equations are solved using the open-source Computational Fluid Dynamics (CFD) library, OpenFOAM $₫$ [28]. OpenFOAM $₫$ has the capability to numerically solve coupled, non-linear differential equations in both space and time on arbitrary polygonal Two-Dimensional (2D) and Three-

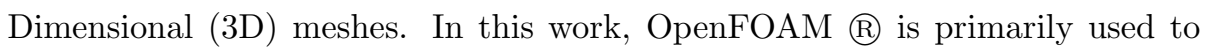
solve the steady, 2D Navier-Stokes equations.

A conventional approach in finite-volume CFD is to use a Neumann boundary condition for pressure on all but one boundary where a Dirichlet condition is used to set the reference pressure. Generally, the Dirichlet condition for pressure is applied to the outlet boundary as shown in Eq. (34), and the Neumann condition in Eq. 35 is used on the inlet boundaries.

$\begin{array}{rlrlrl}p & =0 & & \text { on } & & \partial \Omega_{\mathrm{O}} \\ \frac{\partial p}{\partial n} & =0 & & \text { on } & \partial \Omega_{\mathrm{E}}\end{array}$


However, the zero normal derivative condition is not correct when smaller domain sizes are used. In this case, the Neumann condition for the pressure field is calculated by using Bernoulli's equation to relate the pressure and viscous potential. A two-point finite difference is used to calculate the derivative in the direction normal to the boundary as shown in Eq. (37).

$$
\begin{aligned}
& \frac{\partial p}{\partial n}\left(\mathbf{x}_{f}\right)= \\
& \quad \frac{\rho}{2} \frac{\left|\nabla \varphi\left(\mathbf{x}_{f}\right)\right|^{2}-\left|\nabla \varphi\left(\mathbf{x}_{f}-\sqrt{A_{f}} \cdot \hat{n}\right)\right|^{2}}{\sqrt{A_{f}}} \quad \text { on } \quad \partial \Omega_{\mathrm{E}}
\end{aligned}
$$

Where $\mathbf{x}_{f}$ is the center of a face on the boundary, $A_{f}$ is the area of the face, and the normal vector follows the OpenFOAM $\AA$ convention and points outside of the computational domain.

\subsection{Viscous Potential Sub-Problem}

The numerical implementation of the viscous potential is very similar to the conventional boundary-element method used to solve most velocity potential problems. However, there are a few important modifications that need to be highlighted. As discussed in Section 3.2, the solution to the Laplace equation for the velocity potential can be found by distributing singularities of unknown strength on the boundaries of the fluid domain. The strengths of these singularities are determined by solving the linear system formed by applying the boundary conditions at collocation points and making assumptions about the nature of the flow.

In this work, the total viscous potential is composed of five singularity distributions as well as the freestream potential as shown in Eq. (38).

$$
\varphi=\phi_{V}+\phi_{B S}+\phi_{W S}+\phi_{D}+\mathrm{U}_{\infty} x
$$

Where $\phi_{V}$ is the vortex distribution on the body, $\phi_{B S}$ is the source distribution on the body, $\phi_{W S}$ is the source distribution on the wake surface, and $\phi_{D}$ is the dipole distribution on the wake surface. The reason for this mix of singularity distributions is discussed below. 
The body is discretized with flat panels corresponding to the finite-volume discretization from the viscous flow solver. The vortex distribution on the body is represented using panels of linearly varying strength while the source distribution is represented using constant strength panels. The wake surface is either placed on a straight line originating at the trailing edge and parallel to the $x$-axis or on a streamline beginning at the trailing edge. On the wake surface, the source and dipole panels are constant strength, flat, and coincidentally located. In general, the finite-volume discretization is fine relative to a typical boundary-element discretization, which means the use of flat panels should not be a limiting factor in the numerical solution of the viscous potential. If a higher-order-geometry definition were available, i.e. non-flat panels, there is no foreseeable limitation in their utilization.

The body-boundary condition for the viscous potential is applied to the body surface as shown in Eq. 23) and will be further developed later. However, the body-boundary condition is split into two parts. The linear vortex panels satisfy the inviscid lifting problem, Eq. (39), while the source panels account for the effects of viscosity, Eq. (40).

$$
\begin{array}{rlrlrl}
\frac{\partial \varphi}{\partial n} & =-\int_{0}^{\delta} \frac{\partial w_{t}}{\partial t} \mathrm{dn} & & \text { on } & & \partial \Omega_{\mathrm{B}} \\
\left.+\phi_{D}\right) & =-\mathrm{U}_{\infty} \hat{\mathbf{i}} \cdot \hat{n} & & \text { on } & \partial \Omega_{\mathrm{B}} \\
\frac{\partial \phi_{B S}}{\partial n} & =-\int_{0}^{\delta} \frac{\partial w_{t}}{\partial t} \mathrm{dn} & & \text { on } & \partial \Omega_{\mathrm{B}}
\end{array}
$$

The reason that the body-boundary condition for the viscous potential is satisfied in two parts is because the influence of a constant strength vortex panel (a building block of the linear vortex panel) on itself is zero when applying a condition based on the normal velocity. This means that an individual vortex panel cannot produce a flow through itself and must rely on neighboring panels which would result in a poorly conditioned coefficient matrix.

For each linearly varying strength vortex panel there are two unknowns, 
$\gamma_{0}$ and $\gamma_{1}$. If the vortex strength is enforced to be continuous at the panel end points, then there is effectively only one unknown per interior panel. The resulting algebraic system contains $N_{\text {body }}+1$ unknowns with $N_{\text {body }}$ equations. The Kutta condition or total circulation condition serves as the additional equation to solve for the unknown strengths.

\subsubsection{Circulation condition for the viscous potential}

As described in Section 3.2 .3 the equal-pressure Kutta condition is only used for the inviscid potential while the viscous potential uses the total circulation condition. The equal pressure condition is satisfied by calculating the pressure at two points located behind the trailing edge as shown in Eq. (41). Upon testing, the resulting pressure distribution on the body was found to be insensitive to the exact location of the point.

$$
\mathbf{x}_{E P}^{+/-}=\mathbf{x}_{T E}+l_{\text {panel }} \hat{\mathbf{i}} \pm l_{\text {panel }} \hat{\mathbf{k}}
$$

300 dition is enforced.

The equal pressure condition is a non-linear condition, because of this nonlinearity the equal pressure condition is not satisfied exactly without iteration. An iterative scheme is not employed because the validation of the pressure coinviscid potential is only used as the initial condition for the velocity decomposition approach and has little affect on the final solution.

The total circulation condition uses the Kutta-Joukowski theorem to set the lift on the body in the viscous potential equal to the lift on the body from the Navier-Stokes sub-problem. Eq. 29] is discretized as shown in Eq. 42, Here $\gamma_{m}$ is the average circulation on panel $m$.

$$
\sum_{m=0}^{N_{\text {body }}} \gamma_{m} \cdot l_{\text {panel, } m}=\Gamma^{N S}
$$




\subsubsection{Source and dipole distribution on the wake surface}

On the wake surface, the boundary conditions in Eqs. (30) and (31) are satisfied.

$$
\begin{aligned}
\frac{\partial \phi_{W S}}{\partial n} & =\frac{\chi^{+}+\chi^{-}}{2} & & \text { on } \\
\frac{\partial \phi_{D}}{\partial n} & =-\mathrm{U}_{\infty} \hat{\mathbf{i}} \cdot \hat{n}+\frac{\chi^{+}-\chi^{-}}{2} & & \text { on }
\end{aligned}
$$

While it is possible to apply two different boundary conditions for two separate unknowns at the same location, the result would be that the sum of these conditions is applied (which is exactly what is done with the boundary condition on the body). However, as shown in Eq. (44) the information about the asymmetry is lost.

$$
\begin{aligned}
\frac{\partial}{\partial n}\left(\phi_{W S}+\phi_{D}\right) & =\frac{\chi^{+}+\chi^{-}}{2}+\frac{\chi^{+}-\chi^{-}}{2}-\mathrm{U}_{\infty} \hat{\mathbf{i}} \cdot \hat{n} \\
& =-\mathrm{U}_{\infty} \hat{\mathbf{i}} \cdot \hat{n}+\chi^{+}
\end{aligned}
$$

The solution to this is to prescribe the wake source strength shown in Eq. 45. The dipole strength is solved using the no-penetration condition on the wake, Eq. 46, where the body singularity distributions are included.

$$
\begin{aligned}
\sigma_{W S} & =2 \cdot\left[\frac{\chi^{+}+\chi^{-}}{2}\right] \\
\frac{\partial}{\partial n}\left(\phi_{V}+\phi_{B S}+\phi_{D}\right) & =-\mathrm{U}_{\infty} \hat{\mathbf{i}} \cdot \hat{n}+\frac{\chi^{+}-\chi^{-}}{2} \quad \text { on } \quad \partial \Omega_{\mathrm{W}}
\end{aligned}
$$

Although this is a simplification that will result in a loss of accuracy in the solution, the loss appears to be minimal because of the observation that source panels which lie along a straight line, as in the case of a flat plate, have zero influence on each other and the source strength can simply be expressed as twice the normal velocity specified at the boundary. This is an acceptable assumption to make because the wake surface is also straight, or in the case that it is placed on a streamline, very close to straight.

\subsection{Viscous Potential Inner Iteration}

The main purpose of using velocity decomposition is to decrease the computation time required to solve the Navier-Stokes problem by reducing the size 
of the Navier-Stokes domain. This is achieved by finding a velocity potential which satisfies the Navier-Stokes problem directly outside of the vortical region. This is done by using the viscous potential which is modified to include viscous effects. One of the principle ways of including viscous effects is through the use of the body-boundary condition for the viscous potential, Eq. (23). Clearly, the body boundary condition for the viscous potential is a function of the vortical velocity. This presents a problem because the vortical velocity is calculated as the difference between the total velocity and the viscous potential. Therefore, the viscous potential and vortical velocity are both initially unknown and also are dependent on each other. A straightforward solution to this problem is to use an iterative approach.

The iterative approach given in [23], is identical to that which is used here and is described below. First, a number of equations previously defined are restated for convenience. Eq. 18 is the body-boundary condition for the viscous potential directly derived from substituting the velocity decomposition expression into the non-penetration body-boundary condition. Eq. 23 is the final expression for the body boundary condition for the viscous potential. Eq. 48 is an identity which was used to formulate Eq. (23) and is used to develop the iterative approach here.

$$
\begin{aligned}
& \frac{\partial \varphi}{\partial n}=-\mathbf{w} \cdot \hat{n} \quad \text { on } \\
&=-w_{n}(0) \\
&-\int_{0}^{\delta} \frac{\partial w_{t}}{\partial t} \mathrm{~d} n=w_{n}(0)-w_{n}(\delta) \\
& \frac{\partial \varphi}{\partial n}=-\int_{0}^{\delta} \frac{\partial w_{t}}{\partial t} \mathrm{dn} \quad \text { on } \quad \partial \Omega_{\mathrm{B}} \\
& \text { When calculating the body-boundary condition for the viscous potential, }
\end{aligned}
$$
the total velocity field and inviscid potential are initially known. The approach begins with a vortical velocity field calculated as the difference between the total velocity and the inviscid velocity potential, as shown in Eq. (49). The first 
iteration of the body-boundary condition for the viscous potential is calculated from this vortical velocity field as shown in Eq. 500.

$$
\begin{aligned}
\mathbf{w}^{(0)} & =\mathbf{u}-\nabla \Phi \\
{\frac{\partial \varphi^{(1)}}{\partial n}}^{(1)} & =-\int_{0}^{\delta}{\frac{\partial w_{t}}{\partial t}}^{(0)} \mathrm{dn}
\end{aligned}
$$

Where the superscript is the iteration counter for each loop. Eq. (51) gives the next, and subsequent, iterations where $j$ is the iteration counter.

$$
\frac{\partial \varphi}{\partial n}^{(j)}=-\int_{0}^{\delta}{\frac{\partial w_{t}}{\partial t}}^{(j-1)} \mathrm{dn}
$$

Eq. 48 is now used to relate the integral term to two point values of the vortical velocity as shown in Eq. 52 . This is a great simplification which avoids numerical approximation of the integral term.

$$
\frac{\partial \varphi}{\partial n}^{(j)}=-w_{n}(0)^{(j-1)}+w_{n}(\delta)^{(j-1)}
$$

Where $w_{n}(\delta)^{(j-1)}$ is non-zero because the correct body-boundary condition for the viscous potential has not been determined yet. A final adjustment to Eq. (52) is made by using Eq. (47). Eq. (53) shows that the correction to the body-boundary condition for the viscous potential at each iteration is simply the value of the vortical velocity at the $\delta$-boundary.

$$
\frac{\partial \varphi}{\partial n}^{(j)}=\frac{\partial \varphi}{\partial n}^{(j-1)}+w_{n}(\delta)^{(j-1)}
$$

This scheme results in a viscous potential which satisfies the Navier-Stokes problem outside of the vortical region (the vortical velocity is non-zero only in the vortical regions).

\section{Results}

In this section the velocity decomposition approach is applied to deeply submerged lifting problems. First, the velocity potential implementation is validated. Then, the approach of using source and doublet distributions on the 


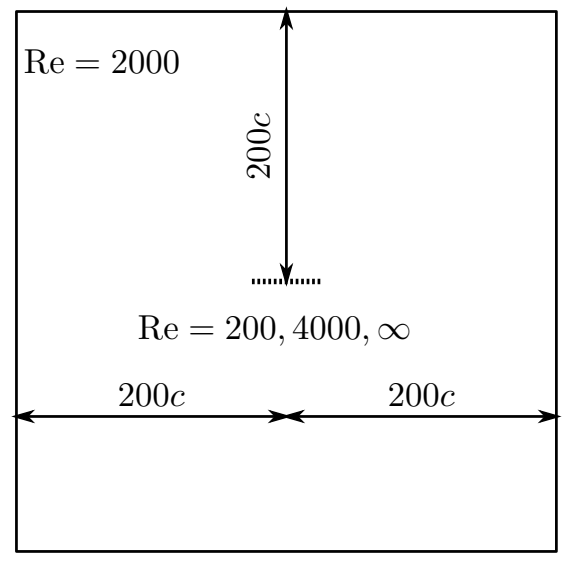

Figure 7: Flat plate Navier-Stokes domain size and Re schematic.

wake surface to capture flow asymmetry is analyzed by applying that idea to the viscous flow over an infinitely thin flat plate with different boundary layer thicknesses above and below the plate, much like a shear flow. After demonstrating the utility of the wake singularity distribution, the full velocity decomposition method is applied to solve for the laminar flow around a NACA0012 foil at $\alpha=5^{\circ}$. Grid convergence studies, including domain size and mesh resolution, are performed. Finally, results are given for the same NACA0012 foil, but at a turbulent $\operatorname{Re}$ of $1.34 \times 10^{6}$ at $\alpha=8^{\circ}$.

\subsection{Flat-plate}

In this section, an asymmetric flow field is constructed to evaluate the algorithm to determine a viscous potential for a problem with lift. The wake surface is comprised of overlapping source and doublet distributions. The asymmetric flow field is generated by the solution to the Navier-Stokes problem over a flat plate. This section will only examine the ability of the source and dipole approach to calculate a viscous potential from an asymmetric viscous flow. The coupled solver is not used.

The plate has a length of $c=1.0 \mathrm{~m}$, is parallel to the $x$-axis and the leading edge is located at $(x, z)=(0,0)$. The free-stream velocity is $\mathrm{U}_{\infty}=1.0 \mathrm{~m} / \mathrm{s}$ and the Re has values of $200,2000,4000$, and $\infty$ that are obtained by changing the 


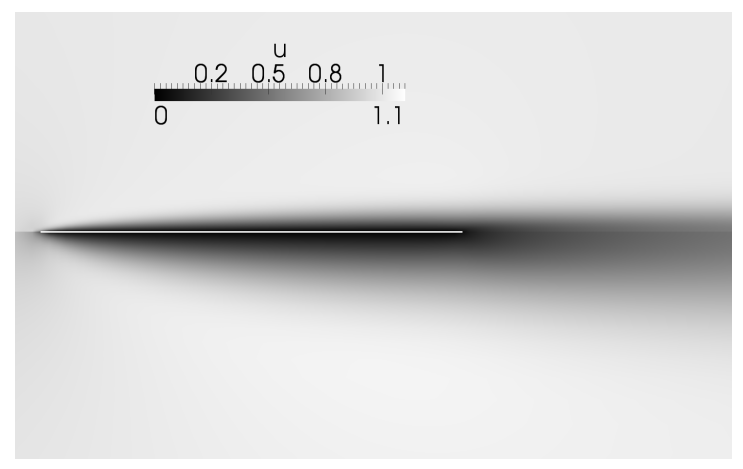

Figure 8: Contour of the streamwise velocity for the Re2000/200 case over the flat plate.

kinematic viscosity. Navier-Stokes solutions for the different Re are generated on large half-domains. The domains were then joined together at $z=0$ where the top and bottom halves are from different Re. An example of this is shown in Figs. 7 and 8 . The top half-domain always has the $\mathrm{Re}=2000$ solution while the 


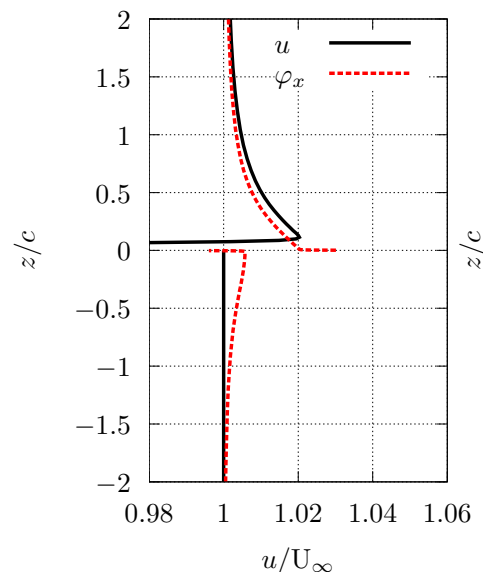

(a) streamwise velocity at the midchord $(x / c=$ $0.5)$

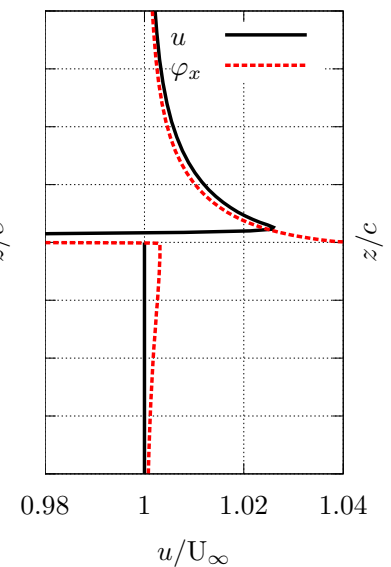

(b) streamwise velocity at the trailing edge $(x / c=1.0)$

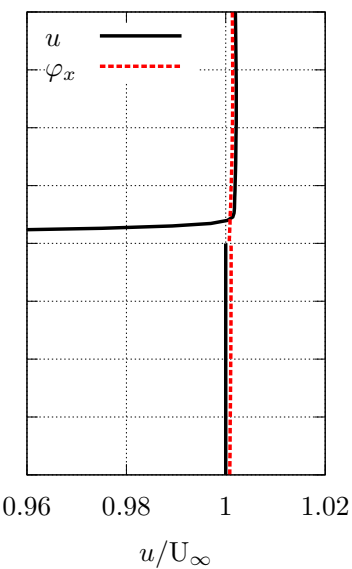

(c) streamwise velocity downstream $(x / c=$ $3.0)$

Figure 9: The streamwise velocity at three sample lines for the $\operatorname{Re} 2000 / \infty$ case.

cases, the strength is the largest on the body and then decays quickly into the wake as the strength of the vorticity decays. In the Navier-Stokes flow, the streamlines constrict slightly near the trailing edge of the body, this is why the source strength is negative directly behind the trailing edge.

The dipole strength, on the other hand, represents the asymmetry of the flow in the vortical region. The dipole strength on the plate is positive for the $\operatorname{Re} 2000 / 4000$ and Re2000/ $\infty$ cases because the vortical layer on the top of the plate is thicker than the bottom and requires flow through the plate from the bottom to top to displace the streamlines more. The opposite is the case in the Re2000/200 case where the thicker vortical layer is on the bottom. This requires flow through the plate from the top to the bottom of the plate.

\subsection{Laminar NACA0012}

This section demonstrates the ability of the velocity decomposition approach to considerably reduce the domain size of the Navier-Stokes sub-problem while maintaining accuracy in the measured quantities of $C_{L}, C_{D}$, and $R M S_{\text {error }}$ 


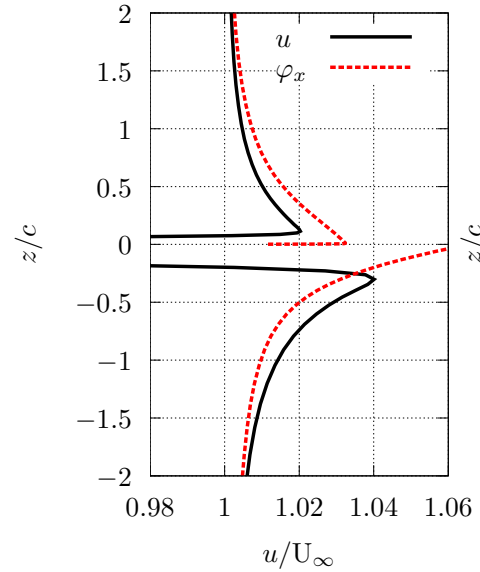

(a) streamwise velocity at the midchord $(x / c=$ $0.5)$

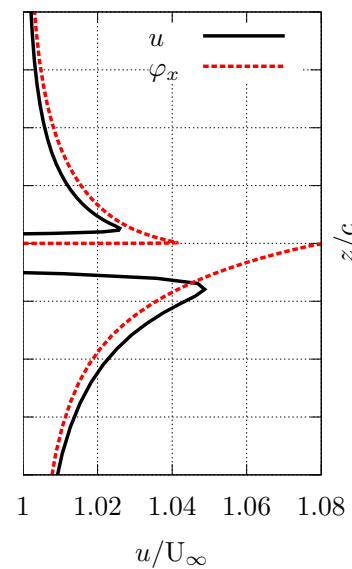

(b) streamwise velocity at the trailing edge $(x / c=1.0)$

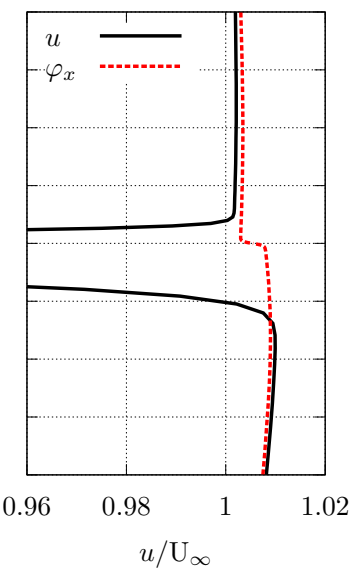

(c) streamwise velocity downstream $(x / c=$ $3.0)$

Figure 10: The streamwise velocity at three sample lines for the Re2000/200 case.

in streamwise velocity as defined in Eq. (54). Results are presented for a NACA0012 foil at $\alpha=5^{\circ}$ and $\operatorname{Re}=2000$. The Navier-Stokes solution on the domain with $x_{\mathrm{E}} / c=2000$ is used as the baseline solution for comparison. Three different results are compared: the Navier-Stokes solution on a reduced domain using the free-stream velocity as the boundary condition on the inlet boundary $\partial \Omega_{I}$ (see Fig. 5.2 for boundary definitions), the Navier-Stokes solution on the reduced domain using the inviscid potential as a boundary condition for the velocity on inlet boundary, and the velocity decomposition solver on the reduced domain that uses the viscous potential for the boundary condition on the inlet boundary. The result with the inviscid potential velocity is sometimes referred to as the "far-field correction" [29] in the literature and is used because it is thought to be a better approximation than simply using the freestream velocity.

$$
R M S_{\mathrm{error}}=\frac{1}{n} \sqrt{\sum_{i=1}^{n}\left(\frac{\varphi_{x, i}-u_{i}^{2000 c}}{u_{i}^{2000 c}}\right)^{2}}
$$




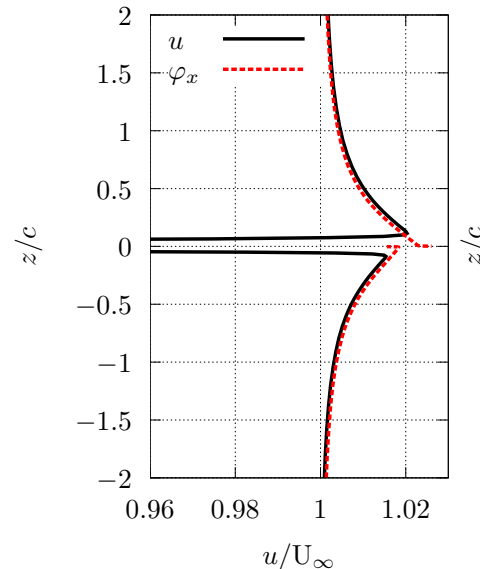

(a) streamwise velocity at the midchord $(x / c=$ $0.5)$

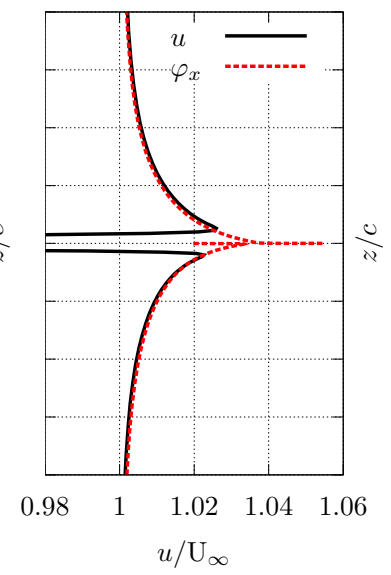

(b) streamwise velocity at the trailing edge $(x / c=1.0)$

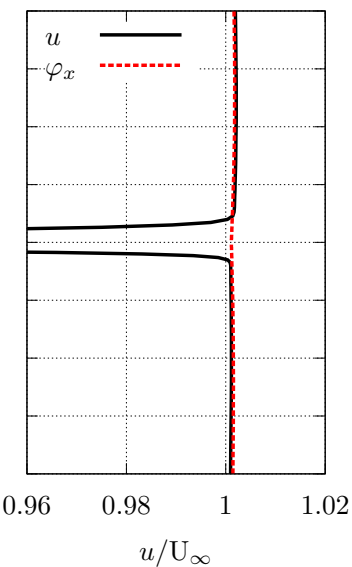

(c) streamwise velocity downstream $(x / c=$ $3.0)$

Figure 11: The streamwise velocity at three sample lines for the Re2000/4000 case.

Fig. 16 shows the error in the lift and drag coefficients compared to the Navier-Stokes solution. The velocity decomposition results give at least an order of magnitude increase (if not better) in accuracy of the measured quantities on a similar sized domain compared to the Navier-Stokes simulations. Also, using the inviscid potential as an improved boundary condition achieves a small improvement in the accuracy, but it is not significant. At this low Re of 2000, the flow is strongly separated (as seen in Fig. 5.2 which results in a large loss of lift. The lift coefficient calculated using velocity decomposition on the domain with boundaries at $x_{\mathrm{E}} / c=2$ is $C_{L}=0.207$ while the inviscid lift coefficient is $C_{L}=0.595$ (for reference, the lift coefficient predicted by the $2 \pi \alpha$ approximation is $\left.C_{L}=0.548\right)$. The use of the inviscid potential as a boundary condition is detrimental because it applies a velocity field that predicts twice the amount of lift present in the real Navier-Stokes solution. The total circulation condition which matches the lift predicted by the viscous potential to the lift present in the Navier-Stokes flow is essential to the accuracy of the velocity decomposition approach in cases of severe loss of lift. 


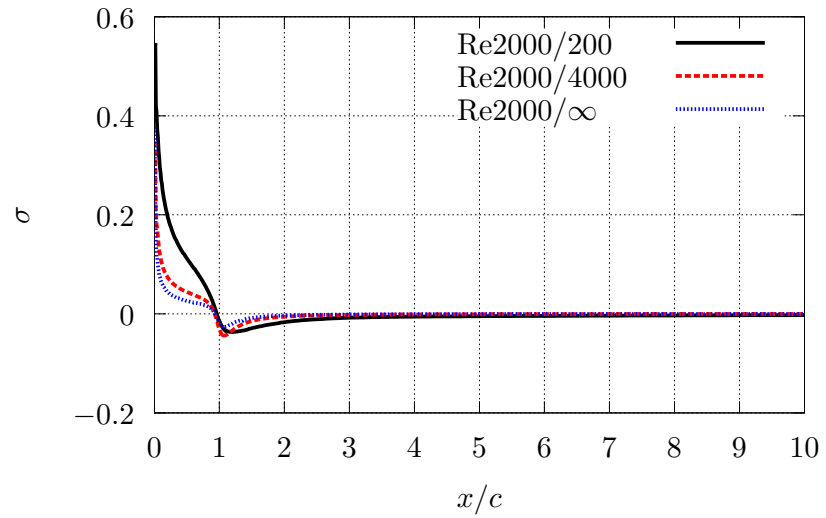

(a)

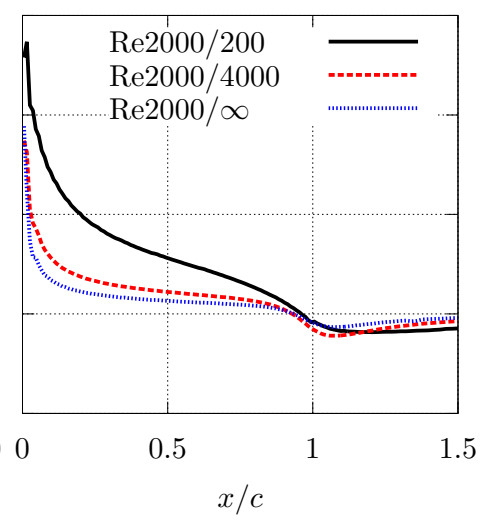

(b)

Figure 12: The value of the source strengths on the body and in the wake for the flat plate at all three Re combinations. The plot on the right has a horizontal axis range that focuses on the region of the plate.

Fig. 17 shows the error in the streamwise velocity calculated using the velocity decomposition approach compared to the Navier-Stokes solution and the Navier-Stokes solution using the inviscid potential for boundary conditions on the total velocity. The velocity decomposition results quickly converge to a high level of accuracy.

Fig. 18 shows contours of the magnitude of the vortical velocity (which is calculated as the difference between the total velocity and the velocity potential). Fig. 18a shows contours of the vortical component calculated using the inviscid potential while Fig. $18 \mathrm{~b}$ shows contours of the vortical component calculated using the viscous potential. These figures demonstrate that the viscous potential drives the vortical component of velocity to zero much more rapidly than the inviscid potential.

Finally, Table 1 compares the lift and drag coefficients as calculated using the large-domain Navier-Stokes solution, the small-domain velocity decomposition solution, and XFOIL [9] in viscous mode. In viscous mode, XFOIL uses a transpiration velocity following the "equivalent source" approach of [7]. Although 


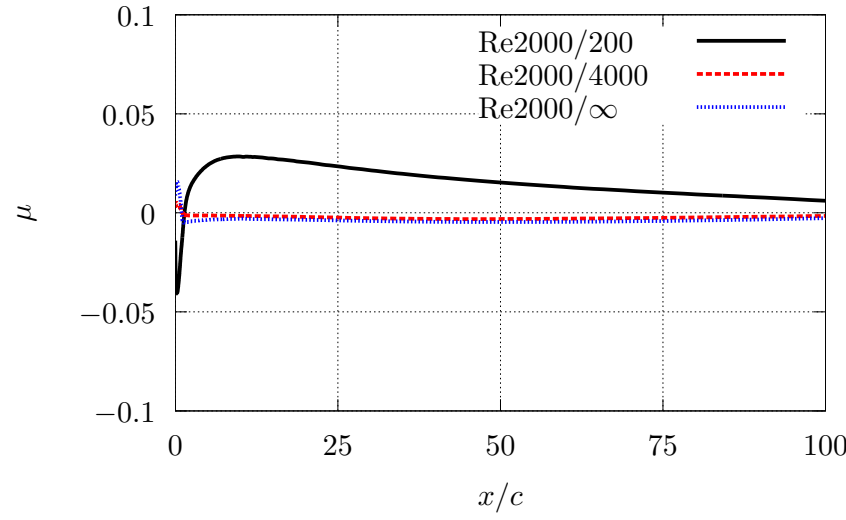

(a)

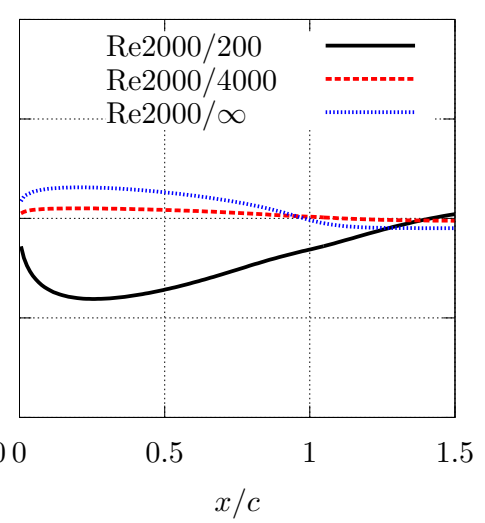

(b)

Figure 13: The value of the dipole strengths on the body and in the wake for the flat plate at all three Re combinations. The plot on the right has a horizontal axis range that focuses on the region of the plate.

Table 1: A comparison of the lift and drag coefficients calculated from the Navier-Stokes solver, the velocity decomposition approach, and XFOIL in viscous mode.

\begin{tabular}{l|c|c} 
& $C_{L}$ & $C_{D}$ \\
\hline Navier-Stokes on $2000 c$ & 0.2064 & 0.0941 \\
Velocity Decomposition on $5 c$ & 0.2065 & 0.0941 \\
XFOIL & 0.2193 & 0.0955
\end{tabular}

XFOIL has a smaller computational expense $(\approx 10 \mathrm{sec})$, the velocity decomposition approach gives a higher accuracy for marginally more cost ( $\approx 1 \mathrm{~min})$, and is suitable for general bodies with massive separation.

\subsubsection{Computational Expense}

One of the benefits of using velocity decomposition is the large computational savings realized. The time savings of using velocity decomposition on the NACA0012 foil case is described in the following tables where three different levels of accuracy are specified. The domain size which provides the specified level of accuracy in the lift, drag and root-mean square error in velocity is reported 


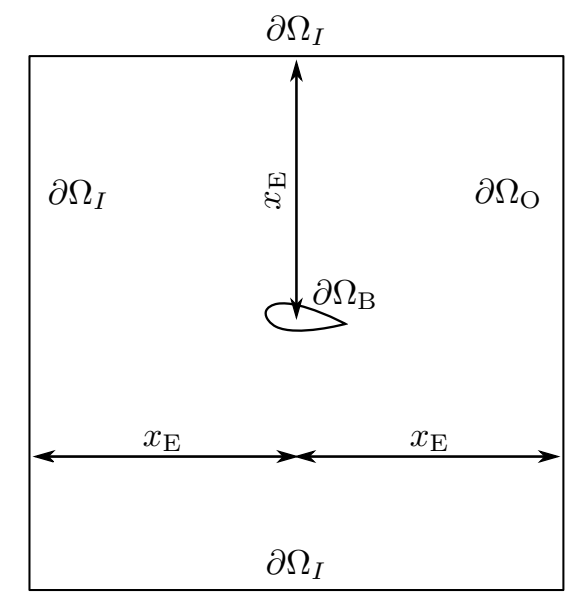

Figure 14: The domain and boundary definitions for the NACA0012 foil simulations.

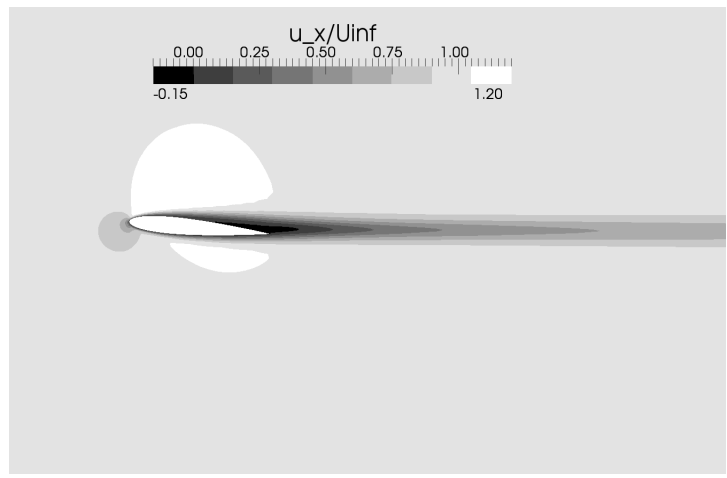

Figure 15: Contour of streamwise velocity for the flow over the NACA0012 at Re $=2000$ and $\alpha=5^{\circ}$. 


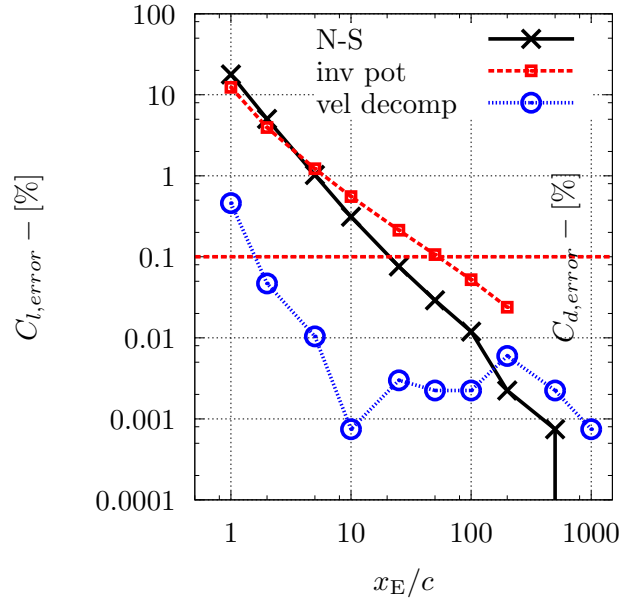

(a) error in the lift coefficient

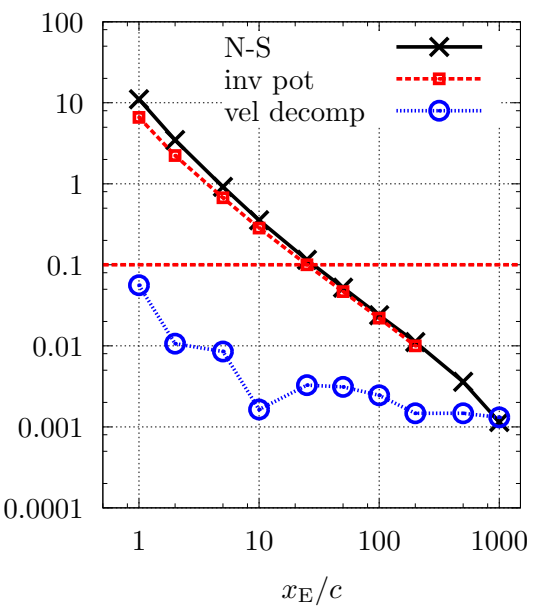

(b) error in the drag coefficient

Figure 16: The error in the integrated forces on the NACA0012 foil calculated using the iterative velocity decomposition solver compared to the forces calculated from a converged Navier-Stokes solution on a large (2000c) domain.

for both the Navier-Stokes solver and the velocity decomposition approach. In order to make a fair comparison, all results were computed on grids which are topologically identical in the overlapping regions and were calculated using a single processor on a multiple-core desktop computer.

In Table 2 the Navier-Stokes domains required to achieve a 1\%, $0.1 \%$, and $0.01 \%$ error threshold are shown. Table 3 shows the same for the velocity decomposition approach. Finally, Table 4 compares the two approaches and the time required to compute the solution which meets that error threshold. The velocity decomposition approach offers a $3-7.5 \times$ speed-up to achieve an equal level of accuracy because of the drastically smaller domains on which the Navier-Stokes sub-problem is solved.

Another observation that can be made is that, for the domain with inlet boundaries at $x_{\mathrm{E}} / c=5$ the difference in computational time is only four seconds. This means that the entire viscous potential calculation (including all five updates and 104 more iterations of the SIMPLE algorithm) required less than 


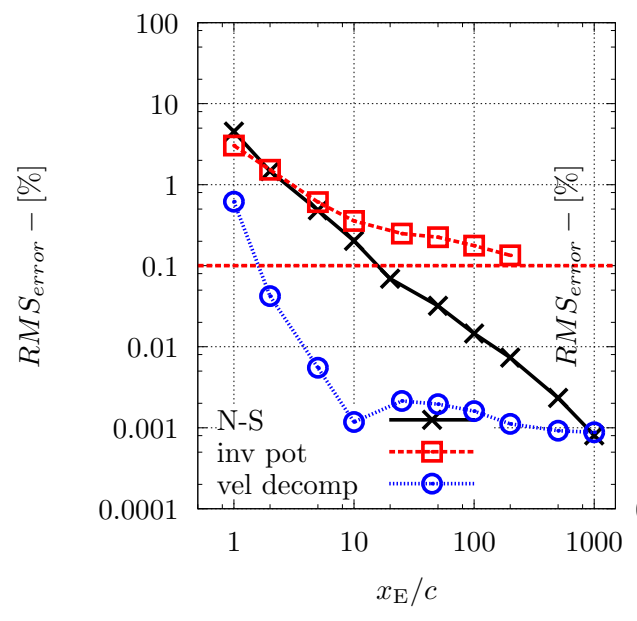

(a) error in the streamwise velocity along a sample line beginning at the midchord

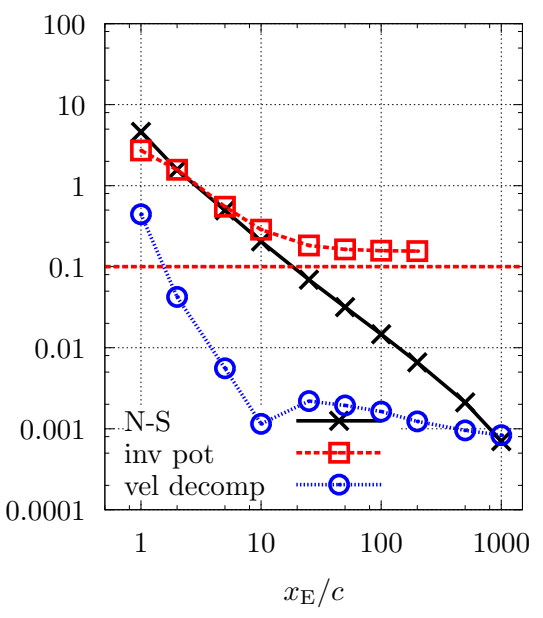

(b) error in the streamwise velocity along a sample line beginning at the trailing edge

Figure 17: Error in the streamwise component of the total velocity calculated using the iterative velocity decomposition solver compared to the streamwise component of the total velocity calculated on a converged Navier-Stokes solution on a large (2000c) domain.
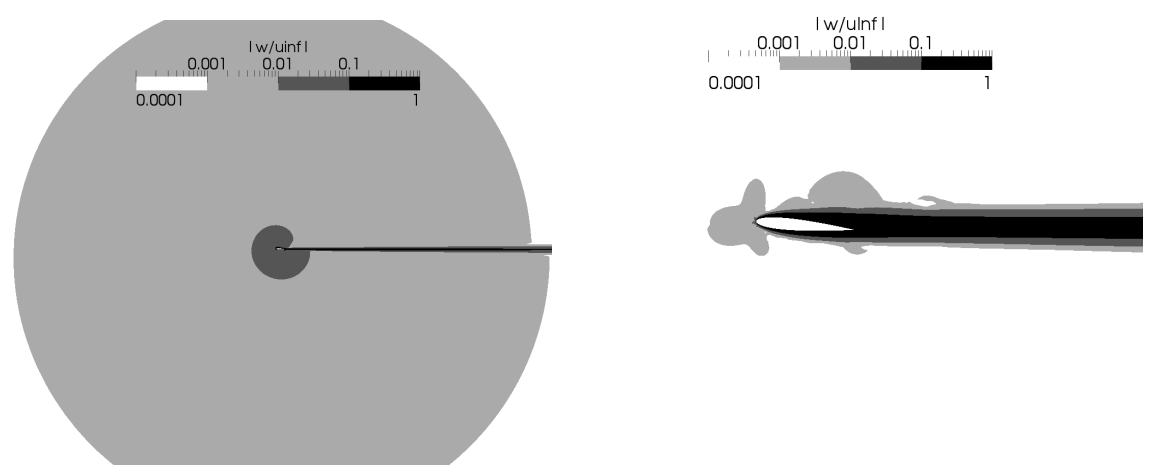

(a) the vortical velocity using the inviscid po- (b) the vortical velocity using the viscous potential

tential

Figure 18: A comparison of the inviscid potential and viscous potential by showing contours of the magnitude of the vortical velocity normalized by the freestream velocity. 
Table 2: Computational time required to converge the Navier-Stokes solver in order to achieve three different orders of accuracy in $C_{L}, C_{D}$, and the streamwise velocity over sample lines at the midchord and trailing edge.

\begin{tabular}{l|c|c|c|c}
$\begin{array}{l}\text { error } \\
\text { threshold }\end{array}$ & $\begin{array}{c}\text { domain size } \\
{[c]}\end{array}$ & $\begin{array}{c}\text { grid points } \\
{[-]}\end{array}$ & $\begin{array}{c}\text { SIMPLE iters } \\
{[-]}\end{array}$ & $\begin{array}{c}\text { time } \\
{[\mathrm{s}]}\end{array}$ \\
\hline $1 \%$ & 5 & 20,182 & 626 & 92 \\
$0.1 \%$ & 50 & 45,262 & 1356 & 338 \\
$0.01 \%$ & 500 & 117,526 & 1859 & 595
\end{tabular}

Table 3: Computational time required to converge the iterative velocity decomposition solver in order to achieve three different orders of accuracy in $C_{L}, C_{D}$, and the streamwise velocity over sample lines at the midchord and trailing edge.

\begin{tabular}{l|c|c|c|c}
$\begin{array}{l}\text { error } \\
\text { threshold }\end{array}$ & $\begin{array}{c}\text { domain size } \\
{[c]}\end{array}$ & $\begin{array}{c}\text { grid points } \\
{[-]}\end{array}$ & $\begin{array}{c}\text { SIMPLE iters } \\
{[-]}\end{array}$ & $\begin{array}{c}\text { time } \\
{[\mathrm{s}]}\end{array}$ \\
\hline $1 \%$ & 1 & 8,800 & 738 & 30 \\
$0.1 \%$ & 2 & 12,398 & 738 & 45 \\
$0.01 \%$ & 5 & 20,182 & 730 & 96
\end{tabular}

four seconds. In other words, for a four second cost in computational time, the use of velocity decomposition will improve the accuracy of the solution by two orders of magnitude in the measured quantities on the domain with $x_{\mathrm{E}} / c=5$.

Furthermore, it is likely that by investigating the effect of the outlet and grid stretching (remove the requirement that the grids are topologically similar in overlapping regions), the speed-up of the velocity decomposition approach could be made even more pronounced.

\subsection{Turbulent NACA0012}

The velocity decomposition approach is now applied to the turbulent NACA0012 foil at $\alpha=8^{\circ}$ and $\operatorname{Re}=1.34 \times 10^{6}$. The velocity decomposition approach is compared to the RANS solutions in the same manner as the laminar case. Fig. 19 
Table 4: A comparison of the time required to converge the velocity decomposition approach to the Navier-Stokes solver.

\begin{tabular}{l|c|c|c}
$\begin{array}{l}\text { error } \\
\text { threshold }\end{array}$ & $\begin{array}{c}\text { N-S } \\
{[\mathrm{s}]}\end{array}$ & $\begin{array}{c}\text { vel decomp } \\
{[\mathrm{s}]}\end{array}$ & $\begin{array}{c}\text { speed-up } \\
{[-]}\end{array}$ \\
\hline $1 \%$ & 92 & 30 & 3.07 \\
$0.1 \%$ & 338 & 45 & 7.50 \\
$0.01 \%$ & 595 & 96 & 6.20
\end{tabular}

shows the error in the lift and drag coefficients compared to the RANS solution calculated on a domain with $x_{\mathrm{E}} / c=2000$. The domain with size $x_{\mathrm{E}} / c=25$ has a predicted drag value equal to the drag predicted by the large domain RANS solution, this is coincidental. Fig. 20 shows the root-mean square error in the streamwise velocity over sample lines at the midchord and trailing edge. For the quantities examined in Fig. 19 and Fig. 20 the error in the velocity decomposition simulations is less than that for the RANS solution on the same size domain. This means that the present method offers improved accuracy for cases in which an external flow must be solved on a necessarily finite domain.

Fig. 21 shows contours of the magnitude of the vortical velocity. Fig. 21a shows contours of the magnitude of vortical velocity calculated using the inviscid potential while Fig. 21b shows contours of the magnitude of the vortical velocity calculated using the viscous potential. The difference is not as pronounced as the laminar case, but still shows the remarkable improvement of the viscous potential over the inviscid potential. These figures show that the viscous potential drives the vortical component of velocity to zero much more rapidly than the inviscid potential. This means that the viscous potential satisfies the Navier-Stokes problem outside of the vortical regions of the flow.

475 The velocity decomposition approach does not give as much advantage in accuracy for the same domain size as it did in the laminar case. It is hypothesized that the larger gradients in the pressure field limit the domain reduction in these high-lift situations. It is doubtful that the calculation of the viscous potential is 


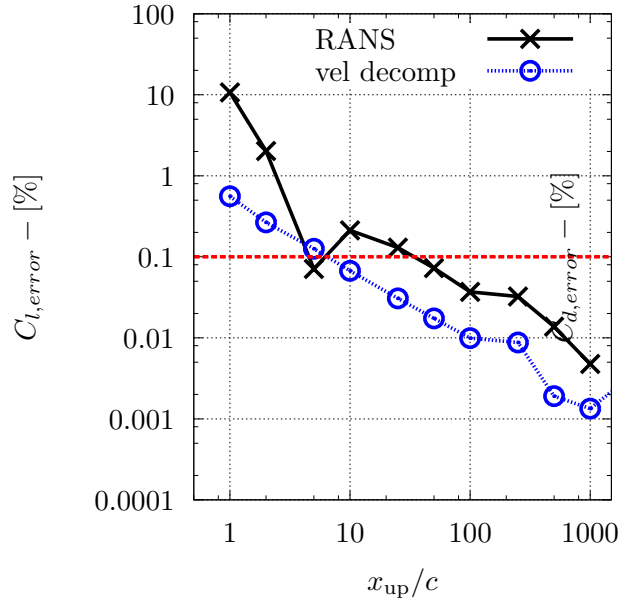

(a) error in the lift coefficient

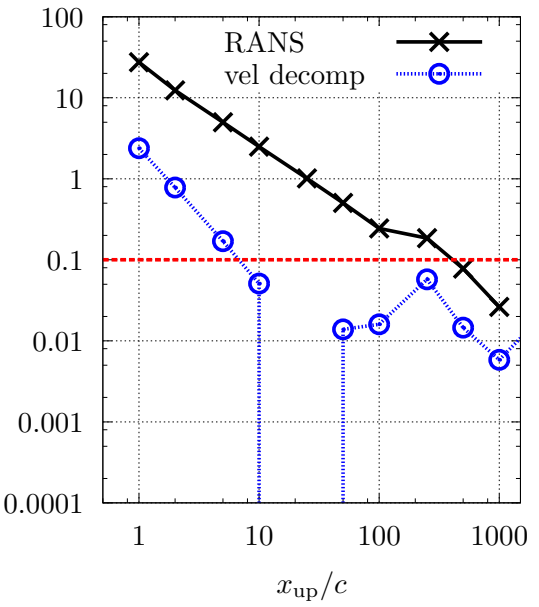

(b) error in the drag coefficient

Figure 19: The error in the integrated forces on the NACA0012 foil calculated using the iterative velocity decomposition solver compared to the forces calculated from a converged Navier-Stokes solution on a large (2000c) domain.

to blame because the viscous potential does satisfy the Navier-Stokes problem outside of the vortical regions (where $\mathbf{w}$ is negligible) to within less than $1 \%$ of the free-stream value as shown in Fig. 21b

\section{Conclusion}

In this work, the velocity decomposition approach is presented for 2D steady lifting problems. Central to this approach is the formulation of the viscous potential for lifting flow. A source and dipole wake distribution is presented with an accompanying condition on the total body-bound circulation. The source and dipole wake distribution allows for the asymmetry in the viscous wake which is present in lifting flows to be included in the viscous potential. The total circulation condition allows for the viscous potential to correctly capture the loss of lift due to separation, which is purely a viscous effect.

The application of velocity decomposition to the flow over a NACA0012 at two different Re's and angles of attack demonstrated the accuracy and efficiency 


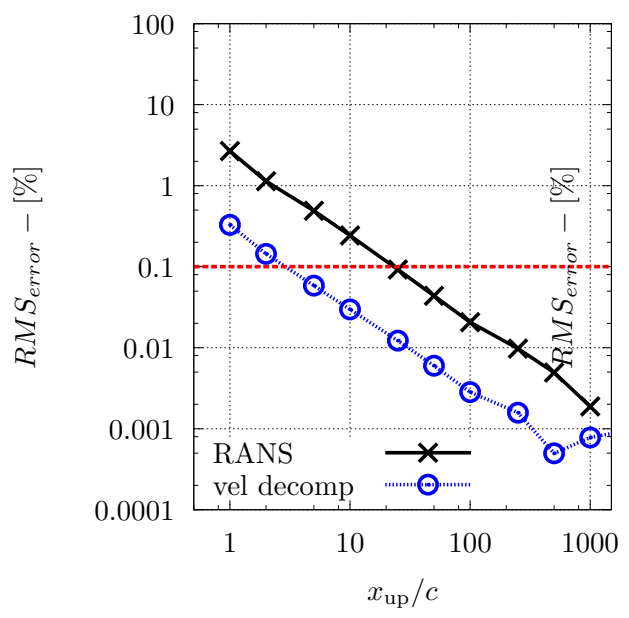

(a) error in the streamwise velocity on a sample line at the midchord

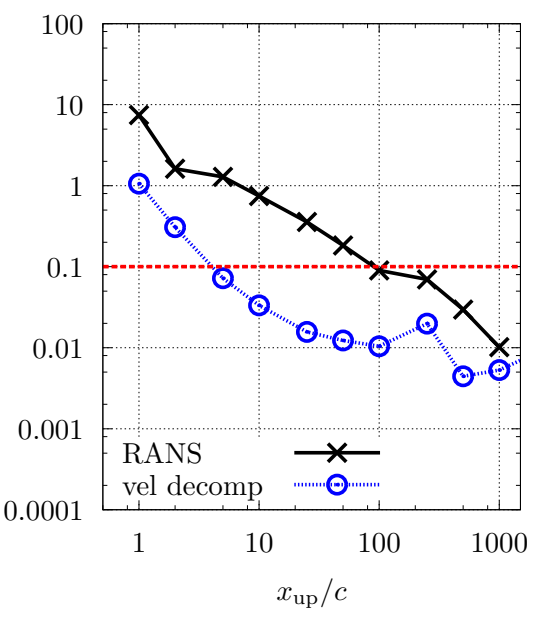

(b) error in the streamwise velocity on a sample line at the trailing edge

Figure 20: Error in the streamwise component of the total velocity calculated using the iterative velocity decomposition solver compared to the streamwise component of the total velocity calculated on a converged Navier-Stokes solution on a large (2000c) domain.
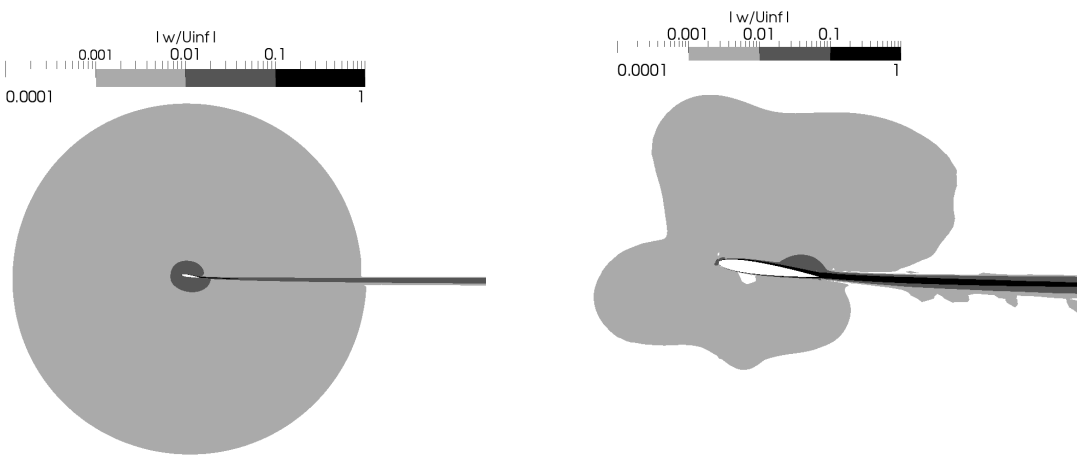

(a) the vortical velocity using the inviscid po- (b) the vortical velocity using the viscous potential tential

Figure 21: A comparison of the inviscid potential and viscous potential by showing contours of the magnitude of the vortical velocity normalized by the freestream velocity. 
of the approach. Specifically, it was demonstrated that the proposed approach is successful in calculating a viscous potential for lifting problems. In the laminar case, the lift and drag coefficients where predicted to within $0.1 \%$ accuracy on a Navier-Stokes domain with $x_{\mathrm{E}} / c=2.0$ using velocity decomposition. The speed-up was $7.5 \times$ compared to the conventional Navier-Stokes solver.

\section{Acknowledgements}

The authors would like to gratefully acknowledge the support of grants from the US Office of Naval Research, Award \#N00014-13-1-0558 under the technical direction of Ms. Kelly Cooper, Award \#N00014-11-1-0484 under the technical direction of Dr. L. Patrick Purtell and Dr. Ki-Han Kim, and Award \#N00014-05-1-0537 under the technical direction of Dr. L. Patrick Purtell and Dr. Linwood Vincent.

\section{References}

[1] D. O. Edmund, K. J. Maki, R. F. Beck, A velocity-decomposition formulation for the incompressible navier-stokes equations, Computational Mechanics (2013) 1-12.

[2] E. Campana, A. Di Mascio, E. Esposito, F. Lalli, Domain decomposition in free surface viscous flows, in: 6th International Conference on Numerical Ship Hydrodynamics, 1994.

[3] E. Campana, A. Di Mascio, P. Esposito, F. Lalli, Viscous-inviscid coupling in free surface ship flows, International Journal for Numerical Methods in Fluids 21 (9) (1995) 699-722.

[4] H.-C. Chen, S.-K. Lee, Interactive RANS/Laplace method for nonlinear free surface flows, Journal of Engineering Mechanics 122. doi:10.1061/ (ASCE) 0733-9399(1996)122:2(153). 
[5] H. Chen, S. Lee, RANS/Laplace calculations of nonlinear waves induced by surface-piercing bodies, Journal of Engineering Mechanics 125 (1999) 1231.

[6] L. Larsson, L. Broberg, K.-J. Kim, Z. D.-H., A method for resistance and flow prediction in ship design, in: SNAME Transactions, Vol. 98, Society of Naval Architects and Marine Engineers, 1990, pp. 495-535.

[7] M. Lighthill, On displacement thickness, Journal of Fluid Mechanics 4 (04) (1958) 383-392.

[8] L. Lemmerman, V. Sonnad, Three-dimensional viscous-inviscid coupling using surface transpiration, Journal of Aircraft 16 (6) (1979) 353-358.

[9] M. Drela, XFOIL: An analysis and design system for low Reynolds number airfoils, in: Low Reynolds Number Aerodynamics, Vol. 54 of Lecture Notes in Engineering, Springer, 1989, pp. 1-12.

[10] L. Morino, Helmholtz decomposition revisited: vorticity generation and trailing edge condition, Computational Mechanics 1 (1) (1986) 65-90.

[11] L. Morino, P. Beauchamp, A new potential-vorticity decomposition for the boundary-element analysis of viscous flows, in: Computational Mechanics 88, Springer, 1988, pp. 13-16.

[12] L. Morino, Toward a unification of potential and viscous aerodynamics: Boundary integral formulation, Applied Mathematics in Aerospace Science and Engineering (1994) 49-79.

[13] L. Morino, F. Salvatore, M. Gennaretti, A new velocity decomposition for viscous flows: Lighthill's equivalent-source method revisited, Computer Methods in Applied Mechanics and Engineering 173 (3-4) (1999) 317-336.

[14] L. Morino, A primitive-variable boundary integral formulation unifying aeroacoustics and aerodynamics, and a natural velocity decomposition for 
vortical fields, International Journal of Aeroacoustics 10 (2) (2011) 295400.

[15] A. Shatalov, M. Hafez, Numerical solutions of incompressible navier-stokes equations using modified bernoulli's law, International journal for numerical methods in fluids 43 (9) (2003) 1107-1137.

[16] M. Hafez, A. Shatalov, E. Wahba, Numerical simulations of incompressible aerodynamic flows using viscous/inviscid interaction procedures, Computer Methods in Applied Mechanics and Engineering 195 (23-24) (2006) 31103127.

[17] M. Hafez, A. Shatalov, M. Nakajima, Improved numerical simulations of incompressible flows based on viscous/inviscid interaction procedures, Computers \& Fluids 36 (10) (2007) 1588-1591.

[18] D. Joseph, Viscous potential flow, Journal of Fluid Mechanics 479 (2003) 191-197.

[19] D. Joseph, Potential flow of viscous fluids: Historical notes, International journal of multiphase flow 32 (3) (2006) 285-310.

[20] D. Joseph, Helmholtz decomposition coupling rotational to irrotational flow of a viscous fluid, Proceedings of the National Academy of Sciences 103 (39) (2006) 14272-14277.

[21] F. Dias, A. Dyachenko, V. Zakharov, Theory of weakly damped free-surface flows: A new formulation based on potential flow solutions, Physics Letters A $372(2008)$ 1297-1302.

[22] K. Kim, A. Sirviente, R. F. Beck, The complementary RANS equations for the simulation of viscous flows, International Journal for Numerical Methods in Fluids 48 (2005) 199-229.

[23] D. Edmund, A velocity decomposition method for efficient numerical computation of steady external flows, Ph.D. thesis, University of Michigan (2012). 
[24] D. O. Edmund, K. J. Maki, R. F. Beck, An improved viscous / inviscid velocity decomposition method, in: International Workshop on Water Waves and Floating Bodies (IWWWFB), Vol. 26, 2011.

[25] G. Arfken, H. Weber, Mathematical Methods for Physicists, 6th Edition, Elsevier Academic Press, 2005.

[26] J. Katz, A. Plotkin, Low-Speed Aerodynamics, McGraw-Hill, Inc., 1991.

[27] W. J. Rosemurgy, K. J. Maki, R. F. Beck, The application of velocity decomposition to airfoil problems, in: International Workshop on Water Waves and Floating Bodies (IWWWFB), Vol. 28, 2013.

[28] H. Weller, G. Tabor, H. Jasak, C. Fureby, A tensorial approach to computational continuum mechanics using object-oriented techniques, Computers in Physics 12 (1998) 620.

[29] J. L. Thomas, M. Salas, Far-field boundary conditions for transonic lifting solutions to the Euler equations, AIAA Journal 24 (7) (1986) 1074-1080. 